\title{
RELAÇÃO ENTRE NÍVEL DE ATIVIDADE FÍSICA E VELOCIDADE DE MARCHA EM IDOSOS CENTENÁRIOS
}

DOI 105902/0102830816055

Artigo Convidado

\author{
Letícia Alves Aguiar \\ Universidade do Estado de Santa Catarina \\ lele_aguiar@hotmail.com \\ Inês Amanda Streit \\ Universidade do Estado de Santa Catarina \\ inesamanda@gmail.com \\ Paula Fabricio Sandreschi \\ Universidade do Estado de Santa Catarina \\ paula.sandreski@gmail.com \\ Artur Rodrigues Fortunato \\ Universidade do Estado de Santa Catarina \\ artur.rfortunato@gmail.com \\ Eduardo Hauser \\ Universidade do Estado de Santa Catarina \\ ehauser07@gmail.com \\ Daniel Rogério Petreça \\ Universidade do Estado de Santa Catarina \\ profdaniel@globomail.com \\ Giovana Zarpellon Mazo \\ Universidade do Estado de Santa Catarina \\ giovana.mazo@udesc.br
}

RESUMO: O objetivo deste estudo foi relacionar o nível de atividade física com a força de preensão manual e velocidade de marcha de idosos centenários. A pesquisa descritiva correlacional, envolveu 21 idosos centenários, com média de idade de 101,8 $\pm 2,1$ anos, residentes em Florianópolis, SC, Brasil, em 2011. Foram avaliadas as seguintes variáveis: nível de atividade física, velocidade da marcha, composição corporal e força de preensão manual. Encontrou-se relação significativa apenas entre o número de passos e a velocidade da marcha. A partir desse estudo conclui-se que a velocidade da marcha se relaciona com o número de passos realizados, em média, na semana.

Palavras-chave: Idosos Centenários. Força de Preensão Manual. Atividade Motora. 


\section{Introdução}

Segundo o relatório das Organizações das Nações Unidas (2011), em 2010, existiam cerca de 292 mil pessoas com 100 anos ou mais de idade no mundo e a previsão é de que em 2050 esse número aumente para 3 milhões. No Brasil, no último censo, foram identificados 24.236 brasileiros com 100 anos ou mais de idade, sendo 16.989 mulheres e 7.247 homens(IBGE, 2010).

Aliado ao aumento da população de centenários no mundo observa-se a realização de pesquisas em diferentes países que buscam identificar os determinantes genéticos da longevidade e traçar o perfil funcional, cognitivo e comportamental destes idosos (ROTT et al., 2001; BECKER et al., 2003; ZENG et al., 2002; STATHAKOS et al., 2005;SUZUKI et al., 2005; PERLS, 2005; MOTOJI, 2006). No entanto, no Brasil a investigação nessa temática ainda é escassa.

Evidências epidemiológicas apontam um decréscimo do nível de atividade física com o aumento da idade cronológica, o que torna o sedentarismo um fator de risco de morbidade e mortalidade durante o processo de envelhecimento (DANAEI et al, 2009; OMS, 2012). No Sul do Brasil, em Florianópolis-SC, um estudo epidemiológico com 875 idosos na faixa de idade entre 60 e 101 anos, de ambos os sexos, verificou que $74,3 \%$ desses idosos não realizavam nenhuma atividade física no tempo de lazer, demonstrando que a inatividade física é evidente na população idosa do município (BENEDETTI; PETROSKI; GONÇALVES, 2004). Além disso, o estudo EPIFLORIPA IDOSO, que avaliou 1656 idosos residentes em Florianópolis-SC mostrou que apenas $42,1 \%$ desses idosos são inativos fisicamente (CORSEUIL et al., 2011).

Outro fator relacionado ao baixo nível de atividade física nos idosos é o declínio da força muscular (ACSM, 2009 Wolfe, 2006). Curb et al. (2006) relacionam a redução nas expressões de força de preensão manual (FPM) com sedentarismo, déficits de massa corporal, problemas de saúde e limitações funcionais em atividades que exigem a participação dos membros superiores e inferiores em idosos. Isso explica em parte, o fato da FPM vir sendo utilizada como indicador de força global e funcionalidade em diversos estudos com idosos (RANTANEN et al., 2003; GERALDES et al., 2008). 
Diante do aumento do número de centenários no Brasil e no mundo e dos indícios de que um envelhecimento fisicamente ativo e a manutenção da força muscular podem predizer um indicativo de longevidade saudável, torna-se importante investigar e correlacionar o nível de atividade física com a força de preensão manual da população centenária. Além disso, a análise destas variáveis poderão subsidiar novos conhecimentos para os profissionais da área da saúde mediando suporte e evidências para uma melhor qualidade de vida dos idosos centenários. Assim, este estudo tem como objetivo verificar a relação entre o nível de atividade física com a força de preensão manual e velocidade de marcha em idosos centenários de Florianópolis, SC, Brasil.

\section{Método}

\section{População e amostra}

Esse estudo descritivo correlacional teve como base populacional 48 idosos centenários, com 100 anos ou mais de idade, residentes no município de Florianópolis, SC, segundo os resultados do mapeamento censitário realizado no ano de 2010 pelo Instituto Brasileiro de Geografia e Estatística (IBGE, 2011).

Para localizar os idosos, verificou-se o setor censitário a que pertenciam, conforme os resultados do mapeamento censitário do IBGE (2011). A partir disso realizou-se uma busca de informações nos Centros de Saúde, através dos agentes comunitários, os quais identificaram os idosos e indicaram o endereço dos mesmos. Outra estratégia utilizada para localizar os idosos centenários foi o contato com líderes de Associações Comunitárias e Centros de Convivência, bem como a divulgação na imprensa impressa e televisiva local e regional.

A amostra foi composta por 21 idosos, conforme organograma abaixo, que ilustra o recrutamento e perda amostral dos idosos centenários participantes deste estudo.

Figura I: Organograma do recrutamento e perda amostral dos idosos centenários participantes deste estudo. 


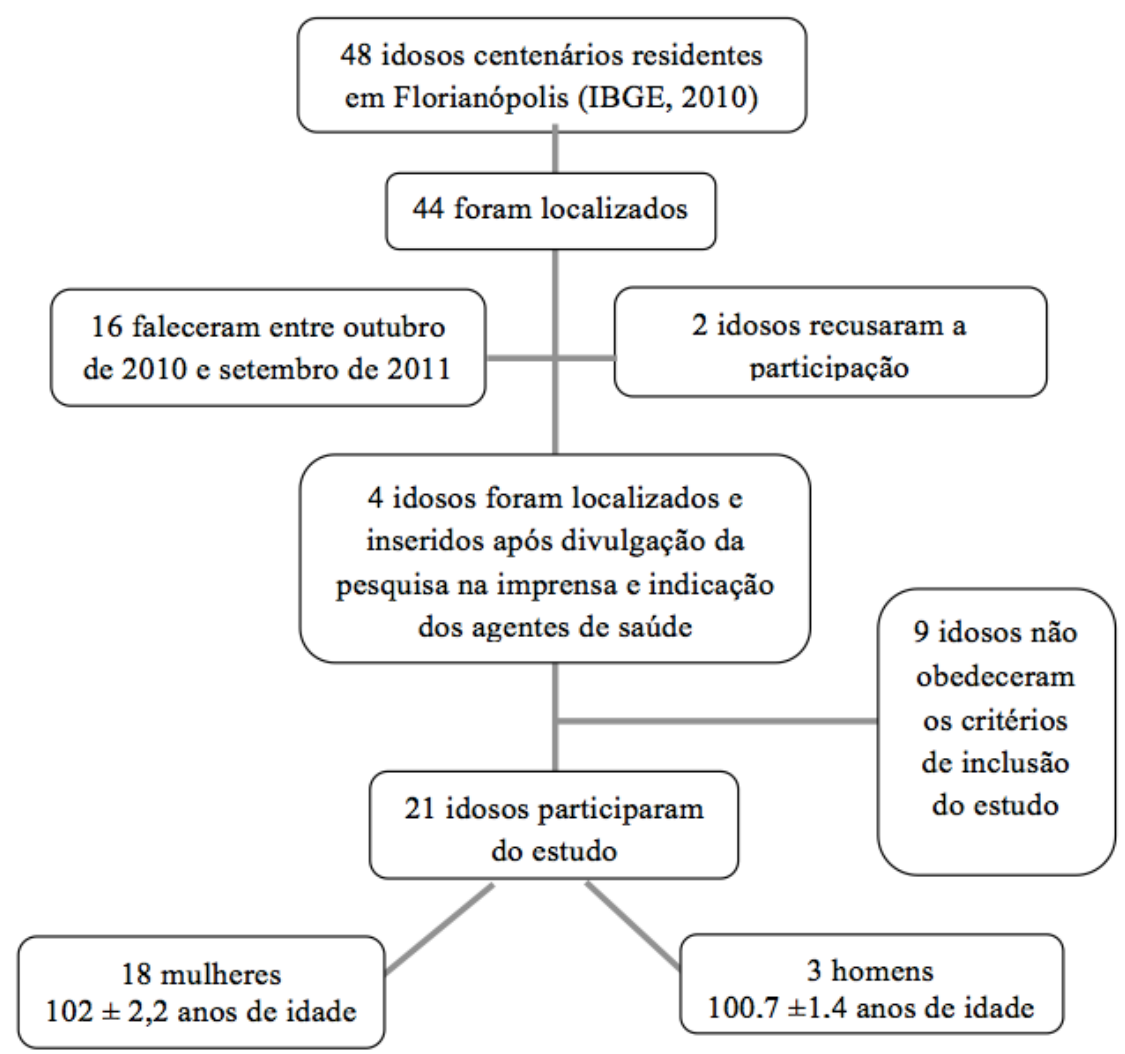

\section{Aspectos Éticos}

O presente estudo foi aprovado pelo Comitê de Ética em Pesquisa da Instituição (Protocolo n 149/2010). Os cuidadores e/ou idosos participantes do estudo assinaram um Termo de Consentimento Livre e Esclarecido, segundo as normas estabelecidas pela resolução 466/12 do Conselho Nacional de Saúde para as pesquisas envolvendo seres humanos.

\section{Critérios de inclusão}

Para o presente estudo adotou-se como critérios de inclusão ter idade igual ou superior a 100 anos de janeiro a dezembro de 2011; residir em Florianópolis há mais de cinco anos; idoso e/ou o cuidador tenha aceitado e autorizado a participação no estudo; realização do teste da velocidade da marcha, uso do pedômetro e realização do teste da força de preensão manual. 


\section{Instrumentos e coleta de dados}

Os instrumentos utilizados para avaliação dos centenários foram os seguintes:

I) Pedômetro da marca POWER WALKER TM Modelo PW-610/611, foi utilizado para mensurar o número de passos dos centenários. Ele grava movimentos de passos em resposta à aceleração do corpo no eixo vertical. Os aparelhos foram programados incluindo o horário de início das coletas (período matutino entre nove e 11 horas ou período vespertino entre 16 e 18 horas) e também de acordo com a massa corporal e amplitude da passada do indivíduo. Previamente a cada período de monitorização, foram repassadas as instruções aos cuidadores e/ou idosos sobre o uso correto de acordo com as especificações do construtor. Para minimizar essa variabilidade intraindividual, estudos (FREEDSON \& MILLER, 2000; TROS et al., 2000) sugerem que a avaliação contemple vários dias consecutivos, incluindo os de finais de semana. Por este motivo, o pedômetro foi utilizado nos idosos centenários durante sete dias consecutivos, durante uma semana habitual. O nível de atividade física foi analisado pormeio da média de passos semanais (7 dias).

II) Dinamômetro da marca SAEHAN® modelo SH5001, foi utilizado para mensurar a força de preensão manual máxima. O procedimento do teste seguiu as recomendações da AmericanSocietyofHandTherapists(ASHT). O avaliador dá um comando verbal em volume alto para início do teste, no qual o idoso deve puxar a alça do dinamômetro com a mão predominante e mantê-la por seis segundos e depois relaxá-la. As medidas são apresentadas em quilograma-força (Kgf). Foram utilizados os dados da preensão manual das duas mãos, embora a mão predominante, para todos os sujeitos participantes do estudo seja a mão direita.

III) Teste de velocidade da marcha avaliou o tempo (em segundos) gasto para percorrer uma distância de 4,6 metros adaptado de Gonçalves (2008). Para iniciar a cronometragem, o idoso foi posicionado no início do percurso e aguardou o comando verbal: "Já!". O final do percurso foi sinalizado pela presença de um avaliador. Foi recomendado ao idoso que utilizasse um calçado usual e, se necessário, poderia ter a companhia de um cuidador ou de dispositivo de auxílio como bengala e andador à marcha. A marcha deveria ser realizada da forma mais rotineira possível. Apenas uma medida foi coletada. Foram adotados os pontos de corte ajustados pelo sexo e altura, conforme proposto por Fried et al. (2001). 


\section{Procedimentos Estatísticos}

Os dados foram analisados no programa estatístico SPSS - StatisticalPackage for Social Sciences - versão 17.0 para Windows. Inicialmente os dados foram tratados por meio da estatística descritiva (média e desvio padrão). Após verificar a distribuição dos dados por meio do teste de Shapiro Wilk utilizou-se o teste de Correlação de Spearman para relacionar as variáveis: número de passos, velocidade de marcha e força de preensão manual. Admitiu-se nível de significância de 5\%.

\section{Resultados}

Conforme a Tabela I, os idosos participantes do estudo apresentaram média de idade de 101,8 $\pm 2,1$ anos e possuíam baixa escolaridade (5,6 \pm 4,4 anos). Além disso, a média do número de passos realizados em sete dias foi de $680,1 \pm 672,2$ e a força de preensão manual mensurada foi de $12,6 \pm 4,9$, para a mão dominante.

Tabela I. Características sociodemográficas, nível de atividade física e força de preensão manual dos idosos centenários residentes na cidade de Florianópolis/SC (N=21).

\begin{tabular}{lc}
\hline \multicolumn{1}{c}{ Variáveis } & Média \pm DP \\
\hline Dados sociodemográficos & $101,8 \pm 2,1$ \\
Idade (anos) & $5,6 \pm 4,4$ \\
Escolaridade (anos) & \\
\hline Nível de Atividade Física & $680,1 \pm 672,2$ \\
Número de passos & $19,5 \pm 17,0$ \\
Velocidade da marcha (s) & \\
\hline Força de preensão manual & $12,6 \pm 4,9$ \\
Direita (Kgf) & $9,8 \pm 6,0$ \\
Esquerda(Kgf) $-(\mathrm{N}=20)$ & \\
\hline
\end{tabular}

DP: desvio padrão

Ao verificar as possíveis correlações entre as variáveis nível de atividade física, velocidade da marcha e força de preensão manual foi encontrada uma correlação significativa entre número de passos e velocidade da marcha $(R h o=-0,7 ; p=0,001 ; R 2=0,3)$, ou seja, quanto maior o número de passos, menor a velocidade da marcha, sendo que $30 \%$ da variabilidade 
na velocidade da marcha é explicada pelo número de passos. O Gráfico 1 evidencia a correlação obtida.

Gráfico I. Correlação obtida entre o número de passos e velocidade da marcha de 21 idosos centenários participantes do estudo.

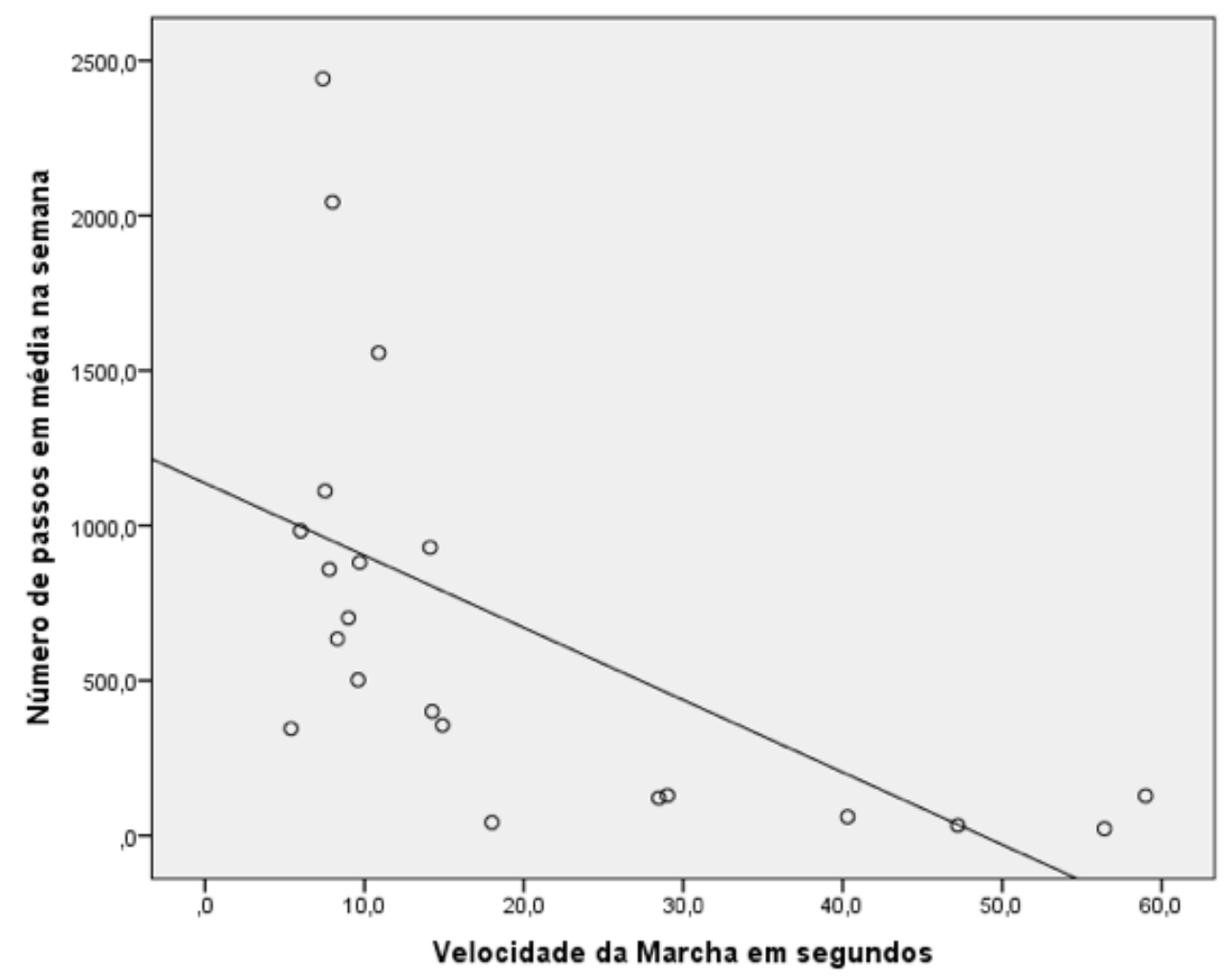

\section{Discussão}

Dos 21 idosos participantes do presente estudo, 18 (86\%) pertenciam ao sexo feminino e três (14\%) ao masculino. Estes dados corroboram aos achados de Ferreira, (2006), Perls; Kunkel e Puca, (2005) e Hagberg e Samuelsson (2008) que evidenciaram maior longevidade entre as mulheres. Neri (2007) descreve tal fenômeno como "feminização do envelhecimento" e ressalva que o mesmo vem acompanhando o envelhecimento populacional no mundo, sendo mais evidente nos estratos etários mais velhos.

Em relação à escolaridade dos centenários observou-se uma média de 5,6 \pm 4,4 anos, semelhante aos estudos de Poon et al. (1991); que ao avaliarem 150 idosos centenários encontraram um baixo nível de escolaridade nesta população. Estes dados também corroboram estudos realizados na China e na Grécia que encontraram baixa escolaridade na população em questão (YAN ZHOU et al., 2010 e STATHAKOS et al., 2005). 
O baixo nível de escolaridade encontrado entre os idosos participantes deste estudo pode estar relacionado com o período de implementação da escolarização na cidade. Segundo os registros do Jornal Diário Catarinense (2011) a escolarização em Florianópolis apenas se iniciou em 1912.

Além disso, para Mazo (1998) a falta de incentivo da família, relacionada ao modelo educacional familiar da época e também às divergências relacionadas aos papeis sociais do sexo feminino e masculino relacionados à essa questão podem ter contribuído para o baixo nível de escolaridade dos idosos centenários.

Ao analisar os dados referentes ao nível de atividade física (número de passos e velocidade da marcha) encontramos uma média baixa para o número de passos realizados na semana, aproximadamente 680,1 passos quando comparado com dados normativos que indicam para populações especiais de 1200 a 8800 passos/dia (TUDOR-LOCKE et al., 2011). Além disso, verificou-se um desvio padrão muito grande (672,2 passos), evidenciando que os idosos centenários são heterogêneos em relação ao número de passos semanais. E com isto conclui-se que, ainda que pouco utilizada, a marcha se encontrava ativa para alguns idosos enquanto para outros se encontrava bastante prejudicada.

Apenas quatro dos 21 centenários realizaram, em média, mais de 1.000 passos por dia. Com relação ao uso de dispositivos para auxílio da marcha, quatro relataram o uso de andador e dois, o uso da cadeira de rodas para evitar quedas, no entanto, deambulavam.

As diretrizes de programas de atividade física para a prevenção de doenças não transmissíveis da Organização Mundial da Saúde (2011) recomendam que os valores referenciais para população adulta saudável seja superior a 10.000 passos por dia. Para idosos saudáveis, com idade entre 60 e 80 anos, recomenda-se entre 6.000 a 8.500 e, para idosos com doenças crônicas, entre 3.500 a 5.000 passos por dia.

Tudor-Locke, Hart e Washington (2009) realizaram uma revisão sistemática sobre o uso de pedômetros como determinante do nível de atividade física em populações idosas, sendo identificados 28 estudos, com amostras com idade entre 50 e 94 anos. Para idosos com 85 anos ou mais, os autores encontraram uma média de 2.015 passos por dia, porém os autores sugeriram ao final do estudo, que 1.000 passos por dia trata-se de um valor referencial coerente para determinar a condição do idoso longevo saudável, uma vez 
que 1.000 passos são equivalentes a uma caminhada rápida de 10 minutos em adultos saudáveis.

Em relação ao tempo gasto para mensurar a velocidade da marcha dos idosos centenários, verificou-se uma média de 19,5 segundos gastos para percorrer 4,6 metros. Observa-se um desvio padrão alto tanto no número de passos como na velocidade da marcha, reforçando novamente o quão a amostra é heterogênea em relação ao nível de atividade física.

O grande desvio padrão encontrado para os níveis de atividade física no presente estudo pode ser justificada também, segundo Shimizu et al (2007), devido à heterogeneidade do processo de envelhecimento influenciada fortemente pelo ambiente em que o idoso está inserido. A quantidade limitada de estudos com idosos centenários também dificultam comparações sólidas e reforçam a necessidade de novos estudos sobre esta temática.

Para Fried et al. (2001) a avaliação da velocidade da marcha relaciona-se com o nível de fragilidade dos idosos centenários. Para este autor a lentidão da marcha pode ser considerada um critério para medir fragilidade nestes idosos. Diante disto, pode-se considerar que os idosos centenários participantes do presente estudo apresentaram alguma fragilidade.

Outro fator que pode explicar o baixo nível de atividade física nos idosos do presente estudo é o declínio da força muscular dos membros inferiores, naturalmente causado pelo envelhecimento, e que pode ter sido um fator limitante primário ou influenciador nas variáveis analisadas. (BUCHNER et al., 1996; JUDGE et al., 1996; BOHANNON et al., 1998; LAMOUREUX et al., 2002; BARRET; SMERDELY et al., 2002; MENZ et al., 2003).

Ao relacionar o nível de atividade física e a velocidade da marcha foi encontrada uma relação significativa, moderada e negativa entre número de passos e velocidade da marcha. Tal relação evidencia que os idosos centenários mesmo que realizem muitos passos, em média, por semana demandaram maior tempo para realizar a tarefa relacionada à velocidade da marcha. É importante salientar que tal relação ainda evidenciou um índice de determinação de $30 \%$ em relação as variáveis citadas acima.

Para Menz; Lord; Fitzpatrick, (2003) os idosos caminham mais lentamente, principalmente, em virtude de uma redução da amplitude das passadas, associada a um 
declínio da flexibilidade de quadril e tornozelos e da força dos músculos flexores do tornozelo e, dos extensores e flexores do joelho. Isso seria de certa forma, compensado pelo aumento da cadência e do tempo da fase de apoio do passo, ainda que com um contato mais curto do calcanhar com o solo.

Nesse sentido, políticas que promovam a prática de atividade física sistematizada e regular nesta população poderiam trazer benefícios nas variáveis relacionadas ao nível de atividade física e força de preensão manual. Alguns estudos já apontam esta prática como promotora da saúde mental e funcional no idoso, promovendo melhor qualidade vida para estes indivíduos (ANTUNES et al., 2006; DESLANDES et al.,2009).

\section{Conclusões}

A partir desse estudo, observa-se uma relação entre o número de passos e a velocidade da marcha de idosos centenários. O nível de atividade física diminuído é uma característica de idosos com idade avançada e, neste contexto intervenções para a manutenção da mobilidade se fazem necessárias. Nesta perspectiva, e considerando que a amostra se evidenciou bastante heterogênea em relação aos dados analisados, são necessárias maiores investigações acerca desta temática. Destaca-se a importância de investigações acerca de idosos centenários para subsidiar políticas públicas preventivas, por meio da capacitação de profissionais da área da saúde, cuidadores familiares e de instituições de longa permanência, servindo como aporte para que os idosos possam sempre ser os maiores beneficiários. 


\section{Referências}

ANSELMI, C.V. et al. Associatio nof the FOXO3A Locus with Extreme Longevity in a Southern Italian Centenarian Study. Rejuvenation Research. v. 12, n. 2, p.124 - 132, 2009.

ANTUNES, H. K. M. et al. Exercício físico e função cognitiva: uma revisão. Revista Brasileira de Medicina do Esporte. v. 12, n. 2, 2006.

BENETTI, M. Z. Estilo de vida de idosos centenários de Florianópolis, SC. Universidade do Estado de Santa Catarina, Programa de pós-graduação em Ciências do Movimento Humano. Dissertação de Mestrado, 2011.

CAI X-Y, et al. Association of Mitochondrial DNA Haplogroups with Exceptional Longevity in a Chinese Population. PlosOne. v. 4, n. 7, p. 1-6, 2009.

CAI X-Y, WANG X-F, LI S-L, QIAN J, QIAN D-G. Association of mitochondrial dnahaplogroups with exceptional longevity in a chinese population. PlosOne. v. 4, n. 7 , 2009.

CAMARANO, A. M. Idosos Brasileiros - Indicadores de Condições de Vida e de Acompanhamento de Políticas. Brasília: Presidência da República/Secretaria Geral de Direitos Humanos/Subsecretaria de promoção e defesa dos direitos humanos, 2006.

CHODZKO-ZAJKO, W. J. et al. American College of Sports Medicine position stand. Exercise and physical activity for older adults. Medicine and Science in Sports and Exercise. v. 41, p. 1510-1530, 2009.

CICCONETTI, P. et al .Lifestyle and cardiovascular aging in centenarians. Archives of Gerontology and Geriatrics. Supplement, v. 8, p. 93-98, 2002.

CORSEUIL, M. W. et al. Perception of environmental obstacles to commuting physical activity in Brazilian elderly. Preventive Medicine. v. 53, n. 4 e 5, p. 289-292, 2011.

CURB, J. D. et al. Performance-based measures of physical function for high-function populations. Journal of the American Geriatrics Society. v. 54, p. 737-742, 2006.

DANAEI G. et al. The preventable causes of death in the United States: comparative risk assessment of dietary, lifestyle, and metabolic risk factors. PloS Med. v. 6, n. 4, p. 1-23, 2009. 
DESLANDES A. et al. Exercise and Mental Health: Many Reasons to Move. Neurophycobiology. v. 59, p. 191-198, 2009.

ENGEL, J.; BLACKWELL, R.; MINIARD, P. Comportamento do consumidor. 9. ed. Rio de Janeiro: LTC, 1995.

FLECK SJ.; KRAEMER WJ. Fundamentos do treinamento de força muscular. Porto Alegre: Artmed; 1999.

FREEDSON, P. S.; MILLER, K. Objective monitoring of physical activity using motion sensors and heart rate. Research Quarterly for Exercise and Sport, v. 71, n. 2, p. 21-29, 2000.

FRIED, L.P.et al. Frailty in older adults: evidence for a phenotype. The Journal of Gerontology, v. 56, n. 3, p. 146-156, 2001.

GERALDES, A. R. et al. A força de preensão manual é boa preditora do desempenho funcional de idosos frágeis: um estudo correlacional múltiplo. Revista Brasileira de Medicina do Esporte. v.14, n.1, p. 12-16, 2008.

GOMES, M.; TURRA, C. M. Quantos são os centenários no Estado de Minas Gerais? Uma estimativa indireta da população com 100 anos e mais, com base no número de óbitos. In: XVI Encontro Nacional de Estudos Populacionais, ABEP, Caxambu, 2008. Anais do XVI Encontro Nacional de Estudos Populacionais, ABEP. Caxambu, Minas Gerais, 2008.

IBGE. Censo Demográfico 2010: Agregado por Setores Censitários dos Resultados do Universo - Disponível em: <http://censo2010.ibge.gov.br/resultados>. Acesso em: 24 mar.2012.

JANNEY, C. Longitudinal Physical Activity Changes in Older Men in the Osteoporotic Fractures in Men Study. Journal of the American Geriatrics Society., v. 58, n, 6, p 11281133, 2010.

KÜCHLER, A. Povoado no Equador tem dez vezes mais pessoas centenárias que média mundial. Disponível em <http://www1.folha.uol.com.br/folha/equilibrio/noticias/ ult263u467278.shtml> Acesso em: 28 mar. 2012.

KUMON, M.T. et al. Centenários no mundo: uma visão panorâmica. Revista Kairós. v. 12, n. 1, p. 213-232, 2009. 
LONGARAI, R. Hábitos pregressos de atividade física em centenários de Porto Alegre. (Mestrado em Gerontologia Biomédica). Programa de Pós Graduaçãoem Biomedicina. Porto Alegre: Pontifícia Universidade Católica do Rio Grande do Sul, 2005.

MENZ, H.B.; LORD, S.R.; FITZPATRICK, R.C. Age-related differences in walking stability. Age Ageing. v. 32, p. 137-142, 2003.

MOTTA, M. et al. Cognitive and functional status in the extreme longevity. Archives of Gerontology and Geriatrics, v. 46, n. 2, p. 245-252, 2008.

NAHAS, M. V. Atividade física, saúde e qualidade de vida: conceitos e sugestões para um estilo de vida ativo. 3 ed. Londrina: Midiograf, 2003.

ORAMAS, J. Cientistas de 13 países com centenários cubanos. Disponível em: <http://www.granma.cu/portugues/2005/mayo/mart24/22club.html. Acesso: 24 Mar2012.

Organização Mundial da Saúde. Envejecimiento Activo: un Marco Político. Revista Espanhola de Geriatrria e Gerontologia; n. 37, p. 74-105, .2002,

Physical Activity Guidelines Advisory Committee: Physical Activity Guidelines Advisory Committee Report, 2008 Washington, D.C.: U.S. Department of Health and Human Services; 2008.

POON, L.W. Health and adaptation styles of centenarians. Gerontologist. v. 31, n. 2, p. 1-6, 1991.

POULAIN, M. Identificação de área geográfica caracterizada pela extrema longevidade na Ilha da Sardenha. Archives of Gerontology and Geriatrics, v. 50, p. 48-50, 2010.

RANTANEN, T. et al. Handgrip strength and cause-specific and total mortality in older disabled women: exploring the mechanism. Journal of the American Geriatrics Society. v. 51. p. $636-641,2003$.

SAWABE, M. et al. Standard organ weights among elderly Japanese who died in hospital, including 50 centenarians. Pathology International. v. 56, p. 315-323, 2006.

SINGH, A. Medicina moderna: rumo à prevenção à cura, ao bem-estar e à longevidade Revista Latino Americana de Psicopatologia Fundamental. v. 13, n. 2, p. 265-282, 2010.

STATHAKOS, D. et al.Assessment of functional health status and life-style characteristics. Experimental Gerontology, v. 40, p. 512-518, 2005. 
TUDOR-LOCKE, C.; HART, T.; WASHINGTON, T. Expected values for pedometerdetermined physical activity in older populations. International Journal of Behavioral Nutrition and Physical Activity. v.6, p. 1-6, 2009.

WILMOTH, J.R. Increase of maximum life-span in Sweden. Science. v. 289, p. 23662368. 2000.

Wolfe, R. R. The underappreciated role of muscle in health and disease. American Journal of Clinical Nutrition. v. 84, p. 475-482,2006.

YATES, L. et al. Exceptional Longevity in Men: modifiable factors associated with survival and function to age 80 years. Archives of Internal Medicine, v. 168, n.3, p. 283-290, 2008. 


\section{RELATIONSHIP BETWEEN LEVEL OF PHYSICAL ACTIVITY AND STRENGTH HANDGRIP OF WALKING SPEED IN ELDERLY CENTENARIANS}

ABSTRACT: The aim of this study was to relate the level of physical activity with the hand grip strength o folder centenarians. A descriptive correlational research involved 21 elderly centenarians, with a mean age of $101.8 \pm 2.1$ years, living in Florianópolis, SC, Brazil, in 2011. The following variables were assessed: physical activity level, gait speed, body composition and handgrip strength. Found a significant relationship between the number of step sand walking speed. From this study it is concluded that the speed of motion relates to the number of steps performed, on average, at week.

Keyword: Centenarians elderly. Gripping Force Manual. Motor Activity.

\section{RELACIÓNENTREELNIVELDEACTIVIDADFÍSICAYLAFUERZADEEMPUÑADURA LA VELOCIDAD AL CAMINAR EN ANCIANOS CENTENARIOS}

Resumen: El objetivo de este estudio fue relacionar el nivel de actividad física con la fuerza de la mano de los centenarios de más edad. Una investigación descriptivo correlacional involucró 21 centenarios ancianos, con una edad media de 101,8 \pm 2,1 años, que viven en Florianópolis, SC, Brasil, en 2011. Se evaluaron las siguientes variables: nivel de actividad física, velocidad de la marcha, la composición corporal y la fuerza de prensión. Encontramos una relación significativa entre el número de pasos y la velocidad de marcha. De este estudio se concluye que la velocidad de movimiento se refiere al número de pasos realizados, en promedio, al semana.

Palabra clave: Centenarios ancianos. Fuerza de empuñadura. Actividad motora. 